\title{
Traduire
}

Une autre perspective sur r tr traduction

Revue française de la traduction

$219 \mid 2008$

Le temps de la réflexion

\section{Du fantastique au gothique : La chute de la maison Usher de Charles Baudelaire}

Ineke Wallaert

\section{(2) OpenEdition \\ 1 Journals}

Édition électronique

URL : http://journals.openedition.org/traduire/877

DOI : 10.4000/traduire.877

ISSN : 2272-9992

Éditeur

Société française des traducteurs

Édition imprimée

Date de publication : 15 décembre 2008

Pagination : 47-72

ISSN : 0395-773X

\section{Référence électronique}

Ineke Wallaert, «Du fantastique au gothique : La chute de la maison Usher de Charles Baudelaire »,

Traduire [En ligne], 219 | 2008, mis en ligne le 15 décembre 2008, consulté le 10 décembre 2020. URL : http://journals.openedition.org/traduire/877 ; DOI : https://doi.org/10.4000/traduire.877 


\title{
Du fantastique au gothique : La chute de la maison Usher de Charles Baudelaire
}

\author{
INEKE WALLAERT
}

\section{Introduction}

Le présent article a une double visée : d'abord, nous voulons réintroduire The Fall of the House of Usher d'Edgar Allan Poe en tant que conte fantastique, en ajoutant quelques contributions au débat sur ce qui distingue le mode fantastique du mode gothique. Ensuite, suivant la méthode critique d'Antoine Berman, nous donnerons une démonstration des transformations que le conte subit dans la traduction de Charles Baudelaire, pour montrer comment l'élément sousjacent de tout discours fantastique, c'est-à-dire l'incertitude et le doute que celui-ci instaure, est affaibli dans la traduction au point que le conte glisse du mode fantastique vers le mode gothique.

Le but de cette étude n'est pas de faire une critique linguistique de la traduction de Baudelaire, ni d'en donner une évaluation en termes de qualité. Nous ne voulons pas davantage réfuter l'opinion de Henri Meschonnic ou d'Antoine Berman selon lesquels Baudelaire fut un "grand traducteur» (Meschonnic 1999 : 54-55, et Berman 1995 : 2). Il convient ici d'affirmer, avec Berman, que c'est le kairos, c'est-à-dire l'arrivée au moment opportun, qui crée les exemples de grandes traductions dans l'histoire, et de confirmer que Baudelaire fut le Kairos de Poe, c'est-à-dire la personnification de cette occasion idéale pour traduire et introduire l'œuvre dans le panthéon de la littérature française(1). Toutefois, nous présentons ici une réécriture faite par

(1) «La grande traduction ne surgit qu' au moment favorable" ». Berman utilise ici le concept de kairos (le moment opportun) comme catégorie temporelle. Nous ajoutons la possibilité de personnifier cette catégorie, en mettant Kairos, cité dans Bailly comme «l'Occasion personnifiée " (Bailly 1950 : 1001). 
Baudelaire qui souligne le caractère historique et personnel de la notion de kairos, et qui démontre que c'est cette historicité spécifique qui fait surgir la nécessité de la retraduction, servant ainsi la survie d'un texte dans une culture cible donnée.

\section{Discours fantastique et discours gothique}

Quand les premiers contes de Poe arrivent en France avec les traductions d'Isabelle Meunier(2), la littérature gothique y est associée à la littérature des bousingots, au romantisme obscur et frénétique, et aux auteurs de la trempe de Pétrus Borel, un auteur que Baudelaire vénérait ${ }^{(3)}$. En même temps, le terme "fantastique " s'était introduit dans le langage courant avec un intérêt grandissant pour l'inconscient et les limites du monde naturel, à partir de 1830 ; Berlioz écrit sa Symphonie fantastique cette même année, et plusieurs auteurs mettent à l'épreuve un nouveau mode d'écriture :

En France, Mérimée, Balzac (L’Église, 1831; Le chef-d'œuvre inconnu, 1832), Th. Gautier (Onuphrius, 1832) sont avec Alexandre Dumas et George Sand les créateurs d'un fantastique original. (Souiller \& Troubetzkoy 1997 : 279)

Une théorisation du fantastique est élaborée par Charles Nodier dans son essai Du fantastique en littérature (1830), et si Nodier n'aborde pas les différences entre la littérature fantastique et gothique comme telles, l'essai montre qu'il était devenu nécessaire de mieux distinguer et séparer deux modes d'écriture qui déjà se croisaient et se mêlaient.

Ce mélange se produit aussi dans le domaine de la littérature anglophone. Il y conduit certains contemporains de Poe, appartenant à

(2) Auteure de quatre traductions publiées dans La démocratie pacifique en 1847 (Richard 1989 : 1584).

(3) Baudelaire écrit une préface à l'édition des Rhapsodies de Pétrus Borel, et en 1861, un chapitre sur celui qu'on nommait le "lycanthrope " dans sa collection "Réflexions sur quelques-uns de mes contemporains " (Cuvres Complètes II 1976 [1861] : 153-156). 
l'establishment littéraire américain qui continuait de vitupérer son œuvre, à qualifier les contes fantastiques de récits gothiques. Cette désignation était rejetée par Poe lui-même, et provenait directement du fait que bon nombre des éléments qui revenaient dans les récits gothiques appartenaient aussi au mode fantastique.

Le roman gothique, quil débouche à la fin sur une explication rationnelle comme chez Ann Radcliffe ou se maintienne dans les cadres du surnaturel comme chez Lewis, repose sur un certain nombre de procédés à effets : châteaux hantés, cimetières, moines inquiétants, apparitions démoniaques de toutes sortes. (Malrieu $1992: 8$ ).

Malrieu ajoute aussi que le fantastique "ne saurait être défini par les ingrédients qui composent les œuvres" (Malrieu 1992 : 18), et nous verrons que cette remarque s'applique parfaitement au récit que nous allons étudier.

Ainsi il y avait, bien avant le moment où Baudelaire se mit à traduire Poe, une conscience croissante que le récit fantastique était basé sur un "surplus de sens" (Milner et Pichois $1996: 153$ ), tandis que le récit gothique peut être résumé comme étant la somme des éléments qui le composent. Ce "surplus de sens » fait par ailleurs écho aux undercurrents of meaning que Poe présente comme caractéristiques et appartenant à that class of compositions in which there lies beneath the transparent upper current of meaning an under or suggestive one (Poe in Thompson 1984 [1840] : 333-341).

Le "surplus de sens " de Milner et Pichois doit ici être élaboré à un niveau plus fondamental, et les différentes stratégies d'écriture qui sous-tendent les modes fantastique et gothique doivent être appréciées plus pleinement. La plupart des auteurs, y compris Rosemary Jackson et Neil Cromwell qui ont élaboré et révisé la description structuraliste que Todorov donne du fantastique, sont en accord avec ce dernier sur le fait que "Le fantastique, c'est l'hésitation éprouvée par un être qui ne connaît que les lois naturelles, face à un événement en apparence surnaturel " (Todorov $1970: 29$ ) - affirmation dont Todorov luimême avoue qu'elle n'est aucunement originale. Un approfondissement de la question produit chez Todorov la remarque suivante : 
"J'en vins presque à croire " : voilà la formule qui résume l'esprit du fantastique. La foi absolue comme l'incrédulité totale nous mèneraient hors du fantastique ; c'est l'hésitation qui lui donne vie. (Todorov 1970 : 35)

Todorov implique par cela que les protagonistes des récits fantastiques se trouvent dans un état de perplexité et de questionnement sur la réalité des choses et des événements qu'ils observent. Ceci est en contraste avec la littérature gothique, où le narrateur et les autres personnages sont parties intégrantes et même prenantes des forces étranges qui les entourent, et invitent le lecteur à suivre le récit sans mettre en doute la nature de leur expérience. Dans la littérature fantastique, au contraire, l'incertitude, souvent vécue par le narrateur au premier chef, contraint le lecteur à l'hésitation entre des explications d'ordre naturel ou rationnel, et une acceptation du surnaturel comme cadre du récit, ce qui, comme l'indique Cromwell, constitue aussi la caractéristique principale attribuée à la littérature fantastique au-delà de la description todorovienne (Cromwell 1990 : 4).

\section{Le discours fantastique comme acte positionnel antithétique}

Pour expliciter cette distinction il semble utile ici de recourir aux notions présentées par Sartre dans son essai L'Imaginaire (Sartre 1940/1986) et de contredire ainsi l'interprétation qu'en donne Bessière, qui applique ces notions aux récits merveilleux (Bessière 1974 : 36-38). Voici le passage dans lequel Sartre présente les façons dont une conscience (au sens du terme allemand Bewusstsein) pose son objet :

Toute conscience pose son objet, mais chacune à sa manière. La perception, par exemple, pose son objet comme existant. L'image enferme, elle aussi, un acte de croyance ou acte positionnel. Cet acte peut prendre quatre formes et quatre seulement : il peut poser l'objet comme inexistant, ou comme absent, ou comme existant ailleurs; il peut aussi se "neutraliser", c'est-à-dire ne pas poser son objet comme existant. Deux 
de ces actes sont des négations, le quatrième correspond à une suspension ou une neutralisation de la thèse. (Sartre 1940/1986:32 - mes soulignements ajoutés)

Contrairement à l'interprétation de Bessière, répétée par ailleurs dans Souiller \& Troubetzkoy (p. 277-278), nous affirmons ici que le récit merveilleux pose bel et bien une thèse, notamment celle qui positionne l'objet comme inexistant, et qu'il doit donc être considéré comme thétique. Traçant ensuite le parallèle avec le récit gothique, nous affirmons que celui-ci, tout comme le récit merveilleux, pose clairement la thèse que l'image qu'il présente est surnaturelle ou irréelle - l'inexistence est la thèse par laquelle le gothique invite l'incrédulité absolue, et les récits gothiques peuvent donc être catégorisées comme thétiques. Au contraire, la littérature fantastique nous impose la quatrième catégorie des actes positionnels de Sartre, celle où le lecteur est invité à une suspension ou une neutralisation de la thèse (et non de l'acte positionnel), et nous pouvons ainsi appeler ces récits non-thétiques ou anti-thétiques. En d'autres termes, le discours fantastique, avec son narrateur envahi par le doute, est anti-thétique puisqu'il suspend la thèse, soit de l'existence ou de l'inexistence des événements. La raison pour laquelle le récit fantastique " tient " est que, malgré le fait que la thèse est suspendue, "cette suspension de la croyance demeure un acte positionnel." (Sartre 1940 : 32 - note de bas de page). Ainsi, tandis que les récits gothiques ne se soucient pas d'établir un cadre potentiellement réaliste, et présentent un monde nettement fictif dont les événements étranges et les personnages font partie intégrante, la littérature fantastique oscille entre la possibilité et l'impossibilité de donner une explication réaliste aux événements, une technique qui entraîne l'ambiguïté et l'incertitude chez le lecteur. En effet, « le fantastique frappe le discours littéraire lui-même d'improbabilité » (Souiller \& Troubetzkoy 1997 : 277-278).

\section{Les arabesques de Poe}

En ce qui concerne ses contes dans les modes " arabesque " et " grotesque ", Poe lui-même refusait à toute force l'étiquette d'auteur 
gothique, ce qui apparaît très clairement dans sa réponse à ceux qui trouvaient ses contes trop " germaniques " pour le goût américain. En effet, Poe confirme dans sa préface des Tales of the Grotesque and the Arabesque où figure Usher que ses contes sont basés sur les mêmes procédés que ceux abordés par Coleridge dans sa Biographia Literaria, et ne peuvent être catalogués comme des récits gothiques :

... it is this prevalence of the "Arabesque(4)" in my serious tales, which has induced one or two critics to tax me, in all friendliness, with what they have been pleased to term "Germanism" and gloom. [...] My friends would be quite as wise in taxing an astronomer with too much astronomy, or an ethical author with treating too largely of morals. [...] If in many of my productions terror has been the thesis, I maintain that terror is not of Germany, but of the soul - that I have deduced this terror only from its legitimate sources, and urged it only to its legitimate results. (in Mabbott 1978 : 473)

Le fait que Poe utilise ici le terme thesis ne remet pas en question ce qui vient d'être exposé sur la nature anti-thétique du récit fantastique : la terreur est la " thèse " de certains contes de Poe, mais les causes de cette terreur restent inexpliquées. Avec ses legitimate sources Poe fait référence à la suspension du jugement de la part du lecteur et du narrateur. En effet, chez Poe la perception incertaine du lecteur avec l'instabilité ontologique des événements qui peuplent ses récits, est souvent générée par un narrateur qui les observe dans un état d'aliénation mentale (sous hypnose, en état d'hystérie, de somnambulisme, d'hallucination, etc.).

Poe infirme donc résolument l'allégation de gothisme pour les Tales of the Grotesque and the Arabesque, tout en indiquant que le mode " arabesque " se rapporte aux techniques qui soutiennent l'unité de l'effet dans ces contes. Avec son allusion à l'astronome taxé de faire trop d'astronomie, Poe met le doigt sur la distinction entre le gothique et

(4) A.-H. Quinn signale que Poe avait trouvé les termes arabesque et grotesque dans l'essai de Sir Walter Scott intitulé "On the Supernatural in Fictitious Composition" (Quinn 1997: 289). 
le fantastique que nous avons faite au début de la présente discussion : le récit gothique est la somme de ses composants, tandis que le récit fantastique utilise souvent les mêmes éléments que le gothique (du moins à l'époque de Poe), mais ne les met pas en jeu de la même façon ni avec le même effet. Nous allons maintenant voir comment Baudelaire a traité cette mise en jeu dans sa traduction.

\section{Méthode critique d'Antoine Berman}

Nous allons aborder la traduction suivant la méthode élaborée par Antoine Berman dans Pour une critique des traductions : John Donne (1995), où ce spécialiste de la traduction présente ce que nous avons nommé une approche para-textuelle des traductions, et que nous jugeons à ce jour la plus complète des méthodes de critique des traductions littéraires. Berman insiste sur l'importance de mener une série d'investigations pré-analytiques bien avant de commencer la comparaison des textes source et cible. Nous en donnons ici les lignes principales, avant de l'appliquer ensuite à La chute de la maison Usher et son texte source.

Avant d'entamer l'étude analytique et comparative d'une traduction, Berman nous demande d'enquêter dans le monde du "sujet traduisant" (Berman 1995 : 16). Ces enquêtes comprennent des informations sur l'horizon traductif et littéraire du traducteur, sa compétence linguistique, sa position générale de traducteur, ainsi que son projet spécifique pour la traduction en question. Il est évident que ces recherches préalables sont indispensables dans le cas de Baudelaire, et que ces informations ne peuvent pas toutes être données ici, puisqu'elles remplissent à elles-mêmes un tome entier. Nous nous limitons à affirmer que Baudelaire avait une position langagière et littéraire très particulière, et un projet de traduction et d'appropriation très personnel.

Berman nous demande ensuite de réaliser une pré-lecture de la traduction et du texte source, et insiste sur les précédents de la traduction à ce stade, puisqu'il faut déterminer les impressions générées par une 
lecture de la traduction et du texte source, en tant que textes indépendants. Il est évident que cette lecture ne peut être que subjective, ce qui est admis de tous, mais reste sans grande importance pour le projet critique : le but de cette étape transitoire est de former des hypothèses qui servent à formuler certaines questions que le critique peut " poser " à la traduction. Nous avançons donc dans le sens inverse des approches à base de corpus, qui étudient le style d'un traducteur, prétendant pouvoir déceler celui-ci en comptabilisant son usage de certaines parties du discours présélectionnées sur des critères horstexte. Que de telles analyses, qui se refusent à une lecture préalable du texte, aboutissent à des résultats peu satisfaisants, et restent loin de comprendre ou de décrire ce qu'ils définissent comme le "style " des traducteurs, ne devrait alors pas nous surprendre (voir, par exemple, Winters 2007, et Wallaert à paraître en 2009).

La pré-lecture sur laquelle Berman insiste aide le " critique " à former des hypothèses à investiguer, et l'analyse comparative qui la suit vise à identifier dans le détail les passages de la traduction où le contenu thématique et les effets different du texte source. Nous devons ici nous limiter à une sélection de ces hypothèses générées par la pré-lecture des deux textes, et nous avons bien entendu sélectionné les éléments les plus éloquents. Il reste à donner les informations bibliographiques sur les textes source et cible et leurs para-textes.

\section{La chute de la maison Usher, The Fall of the House of Usher et leurs épitextes publics}

La chute de la maison Usher de Baudelaire parut d'abord dans Le pays en février 1855 (Richard 1989 : 1348), et fut ensuite reproduit dans le deuxième volume de traductions, les Nouvelles histoires extraordinaires (1857) (Le Dantec 1951 : 337-357). Le texte de Poe parut dans la collection Tales of the Grotesque and the Arabesque en 1840, et ce qui frappe tout d'abord, c'est que Baudelaire n'a retenu aucun élément de ce titre pourtant révélateur des intentions de Poe. Le titre de sa collection où figure une bonne partie des Tales fait simplement écho 
à celui de sa collection précédente (Histoires extraordinaires). Ce point n'est pas sans importance car, comme nous l'avons vu, les termes « arabesque » et " grotesque » constituent les marques grâce auxquelles Poe indique qu'il travaille dans le mode fantastique. Il est aussi important de noter que lors de la publication de ses traductions, Baudelaire avait déjà écrit et publié le texte fondateur pour la réception de Poe en France, c'est-à-dire, Edgar Poe sa vie et ses æeuvres, où Baudelaire pose une fois pour toutes l'image qu'il avait fabriquée pour son écrivain, et par laquelle Poe sera connu en France jusqu’à ce jour.

Il existe une abondance de ce que Genette qualifie d' "épitexte public " en anglais concernant la seule histoire de The Fall of the House of Usher, (Genette 1987/1997 : 316-341), et il existe aussi une bonne quantité d'épitextes sur La chute de la maison Usher - ces derniers étant évidemment basés sur la version française. Les épitextes francophones, comme les commentaires de Marie Bonaparte (1933) ou d'Éveline Pinto (1983), sont pour la plupart obsolètes ou sans intérêt pour le présent article, mais la discussion de Todorov contient un passage clé qui traite de La chute de la maison Usher et qui sera d'importance pour ce qui suit. La lecture de Claude Richard souffre du même mal que ses autres commentaires sur le contenu des écrits de Poe, en ce sens qu'elle repose sur un mélange d'interprétations des textes source et cible (Richard 1989 : 63-65 et 1348-1353 notes). Pour une meilleure compréhension de l'original, nous nous limiterons ici à Kinkead-Weekes (1987), Darell Abel (in Mabott 1978), et Bieganowski (in May 1991).

\section{Pré-lecture : La chute de la maison Usher}

La simulation de ce stade de pré-lecture a été abrégée ici, et nous avons retenu les points qui sont pertinents pour l'analyse suivante, tout en tentant de dessiner l'intrigue du conte pour les lecteurs qui l'ont oubliée.

Dans cette histoire, le narrateur se présente à la première personne et raconte les événements qui conduisent à la dissolution de la lignée et 
de la demeure de la famille Usher. Les deux protagonistes " parlants " sont le narrateur et Roderick Usher, dernier descendant de la lignée, artiste et musicien, et personnage hautement sensible. Les personnages "silencieux " sont Madeline Usher, la sœur jumelle de Roderick, qui meurt au milieu de l'histoire, un médecin et un valet.

Le narrateur, ami d'enfance d'Usher, est mandé à la demeure de ce dernier sans explication précise, et pendant sa résidence s'y trouve de plus en plus affecté par l'atmosphère étrange qui flotte sur l'endroit et ses alentours, et par les circonstances lugubres, d'abord de la mort de Madeline, et ensuite de celle d'Usher. Malgré les changements survenus dans sa condition mentale, le narrateur français semble, tout au long du récit, tenté par des explications rationnelles concernant les phénomènes étranges dont il est le témoin. Pour ce faire, il se repose sur ce qu'il considère être des vérités établies, et sur la connaissance qu'il croit avoir du caractère de son ami. Avec un certain mépris, le narrateur français refuse de se laisser entraîner dans les idées d'ordre surnaturel ou ésotérique auxquelles Usher attribue sa condition, rejetant nerveusement toute interprétation surnaturelle pour s'expliquer l'atmosphère et les événements bizarres, qu'il préfere attribuer à la folie d'Usher et à ses effets contagieux. Si chez le narrateur hésitation il y a, celle-ci vire plutôt au refus d'une quelconque explication ésotérique, et sa façon désespérée de se raccrocher aux interprétations " rationnelles ", ajoutée à la terreur qui l'envahit lentement, conduisent le lecteur à partager son incrédulité, ainsi que son incapacité à juger sa propre condition et à s'expliquer les événements extraordinaires qu'il observe.

Dans les termes sartriens que nous avons adoptés, l'" acte positionnel " par lequel le narrateur choisit de poser les " objets " qu'il perçoit est ici, plus qu'une suspension du positionnement, une manière de " poser l'objet comme inexistant" (Sartre 1940 : 32). Le narrateur se sait et se sent profondément atteint par tout ce qu'il voit et ressent, et se trouve de moins en moins capable de juger sa propre position par rapport aux événements. Cet aspect de son comportement non seulement accroît son absorption dans les événements et la terreur qui 
s'ensuit, mais annule l'importance de ses propres vacillations. Le narrateur est tellement absorbé dans les événements surnaturels qui le font chanceler qu'il ne perçoit plus son propre chancellement.

\section{Pré-lecture : The Fall of the House of Usher}

Chez Poe, le narrateur semble plus enclin à poser les événements d'une façon qui coïncide avec une "suspension, ou une neutralisation de la thèse " (Sartre $1940: 32$ ). Tout en étant aussi atteint au niveau psychologique et physique par les événements, il laisse toutefois place à l'imagination, et son attitude envers les divagations ésotériques d'Usher est moins négative que celle de son binôme français. Limpression que donne la version anglaise du narrateur est celle d'un homme moins nerveux et moins hystérique que celui du texte français. Il regarde les événements avec une perplexité plus curieuse et moins méprisante, et répond aux envolées de sa propre imagination et celle d'Usher avec plus d'intérêt que de rejet. Le narrateur anglais semble donc protégé par ses propres facultés imaginaires contre la terreur qui monte lentement - peut-être parce que ces facultés l'aident à accepter que les causes de cette terreur restent pour lui impénétrables. Quand enfin l'inexplicable a lieu (la sœur morte revient de sa tombe souterraine), le lecteur a deux options : ou bien le narrateur l'entraîne depuis le début dans une hallucination, ou bien il accepte l'explication donnée par le narrateur au début du conte et répétée par Usher, qui suggère que la lignée familiale et la demeure des Usher sont liées par les lois de la sentience. Ces «lois » des correspondances entre le monde organique et inorganique (qui deviendront, d'ailleurs, un thème fondamental dans la poésie de Baudelaire) sont établies dans le conte par un jeu de symétrie, subtilement élaboré par Poe, entre le délabrement de la demeure et le déclin de la lignée. Dans le texte source, tout en rejetant ouvertement l'idée de la sentience comme un caprice de son imagination, une fancy, le narrateur la rumine discrètement et semble plus ouvert aux idées ésotériques de son ami. Le lecteur pour sa part est ainsi plus fortement incité à les contempler, et à tracer leurs repré- 
sentations dans les symboles qui ornent l'histoire et qui constituent un jeu suggestif sous-jacent. La fin ouverte du conte (le narrateur s'enfuit sans donner aucune explication) montre clairement que plusieurs explications sont possibles, et que le lecteur peut suspendre son jugement et méditer sur l'interprétation à donner.

\section{Hypothèses et questions}

Comme le démontre bien l'abondance d'épitextes, plusieurs lectures $\mathrm{du}$ seul texte source sont possibles, et cette variance provient en grande partie du fait que le narrateur oscille lui-même entre l'existant et l'inexistant. Le jeu sur la sentience et la symétrie qui en est l'expression fait l'objet d'une étude plus large, et nous devons ici nous limiter au seul aspect des réactions et des impressions données par le narrateur vis-à-vis des événements étranges de l'histoire.

Les questions qui indiqueront les directions à prendre dans l'analyse qui suit se centreront donc sur le narrateur et ses perceptions :

- Comment le narrateur positionne-t-il ses perceptions et ses émotions, c'est-à-dire, comment voit-il sa propre place dans les événements, et comment les raconte-t-il ?

- Quelles sont les ressources mentales auxquelles il recourt pour juger de ce qui se passe autour de lui ?

- Quelles sont les différences qui peuvent nous donner l'impression que le narrateur français est plus hystérique et moins imaginatif que son binôme anglais?

Nous allons maintenant nous pencher sur les exemples les plus éloquents pouvant nous éclairer sur les sources des impressions laissées par la pré-lecture.

\section{Perceptions, réflexions et sentiments des narrateurs : une comparaison}

L'impression d'un narrateur plus atteint émotionnellement et mentalement est un des points à examiner, et nous citons ici un premier 
passage, qui intervient au début du conte. Le narrateur est arrivé en vue de la maison Usher, et arrête son cheval au bord de l'étang face à la demeure, dont l'aspect extérieur l'interpelle et le dérange. Il se demande :

Qu'était donc ce je ne sais quoi qui m'énervait ainsi en contemplant la maison Usher? C'était un mystère tout à fait insoluble et je ne pouvais lutter contre les pensées ténébreuses qui s'amoncelaient sur moi pendant que jy réfléchissais. (Le Dantec 1951 : 337, lignes 26-31)

What was it - I paused to think - what was it that so unnerved me in the contemplation of the House of Usher? It was a mystery all insoluble; nor could I grapple with the shadowy fancies that crowded upon me as I pondered. (Mabbott $1978: 397-398$, lignes 21-24)

Plusieurs différences sont à observer ici : le rythme de l'anglais est plus calme (Baudelaire omet I paused to think), et au niveau lexical, nous relevons « énervait " pour unnerved, un choix qui donne un narrateur bien plus irrité et nerveux, là où le narrateur anglais est perturbé ou troublé, sans être forcément agité ou nerveux. En contemplant la maison, le narrateur français essaie de "lutter contre " des "pensées ténébreuses, " qu'il veut " combattre " ou auxquelles il veut " résister" (Littré 3 : 3608). Le terme anglais grapple with, to try to deal with (a question, etc.); to try to solve (a problem, etc.) (OED $5: 362$ ), indique une attitude plus contemplative que "lutter ", et de surcroît, le narrateur ne contemple pas des "pensées" mais des fancies.

La différence entre les termes fancy et "pensée " est d'une importance telle pour l'ensemble des traductions de Baudelaire que son examen excède largement la portée de cet article. Comme l'indique Richard, le mot fancy n'as pas d'équivalent direct en français : "Fancy est un idiome anglais. Rêverie, fantaisie, lubie sont peut-être les traductions les moins inexactes" (Richard 1989 : 1302n), et nous ajoutons ici " caprice, ou saut de l'imagination ». Ce terme est en outre lié dans l'œuvre de Poe à des adjectifs comme wild ou mystic, termes que Baudelaire n'a pas non plus traduits selon le sens que Poe leur donnait. 
Les idées de Poe sur la fancy et l'imagination sont étroitement liées à celles de Coleridge sur le sujet(5), et la distinction cruciale entre ces deux facultés mentales implique une distinction de composition poétique que ces facultés tendent à faire naître. Nous savons maintenant que Baudelaire devait connaître les subtilités de cette distinction, puisque l'essai où Poe en parle le plus longuement, The Poetic Principle, fut reproduit par Baudelaire (sans aveu clair) dans ses " Notes Nouvelles sur Edgar Poe», comme le confirme Richard :

On sait que l'essentiel des " Notes nouvelles sur Edgar Poe, " l'article que Baudelaire donna en préface aux Nouvelles histoires extraordinaires (1857), est une traduction non avouée du Principe Poétique. (Richard 1989 : 1463n)

En effet, une comparaison détaillée confirme cette observation, et d'autres ont attiré l'attention sur le fait que Baudelaire reproduit, deux ans plus tard, ces mêmes contenus dans un essai sur Théophile Gautier (1859), remarquant qu'il se cite lui-même, ce qui conduit Patterson à l'exclamation suivante :

Évidemment il est permis de se citer soi-même autant qu'on le désire, mais il est défendu de reproduire les pensées d'un autre auteur en se les attribuant, lors même que ces idées seraient exprimées dans une autre langue. (Patterson 1923 : 85)

Le prestige du géant littéraire qu'est Baudelaire, et la force de l'image de génie accidentel et intoxiqué qu'il avait projetée de Poe, empêchent jusqu'à ce jour les spécialistes francophones de voir et de reconnaître ce plagiat confirmé :

Les " commentaires " dont Baudelaire accompagne ses citations du Poetic Principle ne sont souvent qu'une paraphrase, parfois une traduction littérale du texte de ce morceau... Mais qui oserait prononcer ici le

(5) Poe admirait profondément la pensée de S.T. Coleridge, et plus spécifiquement la Biographia Literaria, où figure la célèbre discussion sur l'imagination conduite par le poète. Poe mène plusieurs discussions sur la différence entre l'imagination et la fancy, qui démontrent sa conscience des courants sous-jacents (underlying currents) qui distinguent les deux modes d'écriture (voir The Poetic Principle, les essais sur Thomas Hood et sur Thomas More, et plusieurs paragraphes et extraits des Marginalia). 
nom de plagiat? Baudelaire, plein de son sujet, reproduit involontairement son auteur et le tient par sa propre substance. (Le Dantec 1951: $1142 n)$

Ne pouvant ici seulement suggérer les conséquences plus larges de la non-traduction que Baudelaire fait du terme fancy, et laissant cette discussion à une autre occasion, nous retournons à notre extrait, où la phrase "pendant que j’y réfléchissais " ajoute un nouveau changement dans les perceptions du narrateur, puisque " réfléchir " indique des activités mentales plus rationnelles et moins rêveuses que ponder. Globalement, l'extrait nous confirme donc l'impression d'un narrateur français qui, au lieu d'avoir recours à son imagination, rejette nerveusement ses propres pensées, là où le narrateur anglais, dans un état troublé mais contemplatif, essaie de comprendre et médite sur les fantaisies que son imagination produit.

Comme l'indique la pré-lecture, plusieurs passages de la traduction nous indiquent que le narrateur se montre trop convaincu de sa propre connaissance a priori des choses. Les lignes suivantes suggèrent qu'il y a, en effet, une différence dans les préparatifs qui ont précédé la visite du narrateur à son ami.

Je m'attendais bien à quelque chose dans ce genre, et j’y avais été préparé non seulement par sa lettre, mais aussi par le souvenir de certains traits de son enfance. (Le Dantec 1951 : 342, lignes 226-228)

For something of this nature I had been prepared, no less by his letter, than by reminiscences of certain boyish traits. (Mabbott 1978 : 402, lignes 173-175)

L'ajout de « je m'attendais bien à " suppose que le narrateur français a pu anticiper et prévoir l'état d'Usher par ses propres moyens. Un exemple crucial de cette supposition apparaît aussi dans la phrase suivante :

Peut-être m'impressionna-t-elle plus fortement, quand il me la montra, parce que, dans le sens intérieur et mystérieux de l'œuvre, je découvris pour la première fois qu'Usher avait pleine conscience de son état, - qu'il sentait que sa sublime raison chancelait sur son trône. (Le Dantec 1951 : 346, lignes 394-398) 
I was, perhaps, the more forcibly impressed with it, as he gave it, because, in the under or mystic current of its meaning, I fancied that I perceived, and for the first time, a full consciousness on the part of Usher, of the tottering of his lofty reason upon her throne. (Mabbott 1978 : 406, lignes 309-314)

Nous avons déjà parlé de la traduction de fancy/fancied, à laquelle se rattache celle de mystic par " mystérieux ", traduction qui indique que Baudelaire avait une interprétation de ces termes différente de celle de Poe. Ce qui frappe surtout dans cet extrait, c'est que le narrateur français n'indique pas qu'il doute de la vérité de ses observations, parlant de " découvrir " quelque chose, là où le narrateur anglais fait une double esquive du positionnement de la thèse avec I fancied that I perceived. Cet exemple nous renvoie à la clé que donne Todorov pour la littérature fantastique, et que nous rappelons ici " "J'en vins presque à croire" : voilà la formule qui résume l'esprit du fantastique. " (Todorov 1970 : 35). En traduisant I fancied that I perceived par « je découvris ", Baudelaire annule l'incertitude du narrateur d'un trait, et omet l'élément de doute qui caractérise, selon Todorov, le fantastique. Todorov, qui, rappelons-le, se basait sur la version baudelairienne pour formuler ses remarques concernant l'œuvre de Poe et, plus précisément, concernant $U$ sher, vient alors involontairement confirmer notre hypothèse selon laquelle le récit change de mode en traduction, par la remarque suivante :

Ainsi donc le fantastique se trouve en définitive exclu de "la Maison Usher ". D'une manière générale, on ne trouve pas dans l'ouvre de Poe de contes fantastiques, au sens strict ... (Todorov 1970 : 54)

Inutile de souligner que Todorov est très seul dans cette perception de la fiction de Poe : en effet la grande majorité des chercheurs qui se sont penchés sur le fantastique mentionnent les contes de Poe, et la plupart des écrits sur Poe décrivent ses contes arabesques et grotesques comme des récits fantastiques. 


\section{Incertitude et ambiguiité}

D'autres incertitudes sont absentes de la traduction. Nous verrons comment Baudelaire a supprimé l'ambiguïté créant la possibilité que Roderick Usher et sa sœur Madeline soient la même personne. En anglais cette ambiguiité est établie par une symétrie subtile et un choix lexical bien précis. La première occurrence se trouve dans le passage suivant la mort de Madeline, où Poe met en scène le narrateur et Roderick :

Nous déposâmes donc notre fardeau funèbre sur des tréteaux dans cette région d'horreur; nous tournâmes un peu de côté le couvercle de la bière qui n'était pas encore vissée, et nous regardâmes la face du cadavre.

(Le Dantec 1951 : 350, lignes 541-544)

Having deposited our mournful burden upon tressels within this region of horror, we partially turned aside the yet unscrewed lid of the coffin, and looked upon the face of the tenant. (Mabbott $1978: 410$, lignes 438-440)

Non seulement Baudelaire accroît l'effet d'horreur par son choix de "funèbre " pour mournful, choix qui augmente légèrement l'atmosphère gothique, mais là où Poe écrit tenant Baudelaire choisit " cadavre". Le choix de Poe n'était pourtant pas anodin : tenant n'est pas un mot qui habituellement décrit des cadavres dans leur cercueil. Il fait ici écho à un passage précédent, où le même terme est utilisé par Poe pour décrire non Madeline, mais Roderick Usher :

Il était dominé par certaines impressions superstitieuses relatives au manoir qu'il habitait, et d'où il n'avait pas osé sortir depuis plusieurs années. (Le Dantec 1951 : 343, lignes 273-276)

He was enchained by certain superstitious impressions in regard to the dwelling which he tenanted, and whence, for many years, he had never ventured forth. (Mabbott 1978 : 403, lignes 213-215)

Baudelaire annule ainsi la possibilité d'amalgame entre Roderick et Madeline, tous deux occupants de la maison qui deviendra aussi leur 
tombe commune. Deux autres extraits confirment que Baudelaire évite toute confusion :

Ce n'était qu'avec peine que je pouvais consentir à admettre l'identité de l'homme placé en face de moi avec le compagnon de mes premières années. (Le Dantec 1951 : 341, lignes 195-197)

It was with difficulty that I could bring myself to admit the identity of the wan being before me with the companion of my early boyhood. (Mabbott 1978 : 401, lignes 150-151)

Mais actuellement, dans la simple exagération du caractère de cette figure, et de l'expression qu'elle présentait actuellement, je doutais de l'homme à qui je parlais. (Le Dantec 1951 : 342, lignes 210-213)

And now, in the mere exaggeration of the prevailing character of these features, and of the expression they were wont to convey, lay so much of change that I doubted to whom I spoke. (Mabbott 1978 : 402, lignes 161-164)

La perte d'ambiguiité et d'incertitude se fait également au travers d'un plus grand engagement émotionnel et personnel du narrateur français. Il semble se comporter avec Usher de façon plus intime, comme un grand frère un peu moralisateur ou pédant. L'extrait suivant est parmi ceux qui tendent à confirmer cette impression :

J'appris ainsi, par intervalles, et par des confidences hachées, des demimots et des sous-entendus, une autre particularité de sa situation morale. (Le Dantec 1951: 343, lignes 271-273)

I learned, moreover, at intervals, and through broken and equivocal hints, another feature of his mental condition. (Mabbott 1978: 403, lignes 211-212)

Le narrateur français n’a pas seulement reçu des " confidences hachées, " il a aussi entendu des " demi-mots " et des "sous-entendus, " là où le narrateur de Poe n'a reçu que des broken hints, qui de surcroît étaient equivocal. En outre, la traduction de mental par " morale " donne un aspect moralisateur au narrateur français qui ne figure pas dans le texte de Poe. Cette intimité accrue et cette attitude un peu pédante, peut être liée à la façon dont Usher a lui-même pré- 
senté sa condition dans un passage antérieur : parlant pour la première fois à son ami de la terreur qu'il ressent, l'Usher de Poe dit I must perish in this deplorable folly (Mabbott 1978 : 403, lignes 201-202), laissant ainsi planer le doute sur les sources potentielles de cette folie, tandis que la version baudelairienne donne " - il faut que je meure de cette déplorable folie»(Le Dantec 1951 : 343, ligne 260), et place ainsi la source de la folie dans la tête d'Usher.

\section{Scepticisme ou imagination ?}

Revenant aux états d'âme de nos deux narrateurs, nous proposons ensuite d'examiner un extrait où le narrateur se voit de plus en plus atteint par l'état d'Usher, et par l'étrangeté des événements. Ici, le narrateur français subit cette influence comme une maladie à laquelle il ne peut pas échapper, ce qui n'est pas du tout le cas pour le narrateur anglais :

Je sentais se glisser en moi, par une gradation lente mais sûre, l'étrange influence de ses superstitions fantastiques et contagieuses.

(Le Dantec 1951 : 351, lignes 583-586)

I felt creeping upon me, by slow yet certain degrees, the wild influence of his own fantastic yet impressive superstitions. (Mabbott 1978 : 411, lignes 470-473)

Nous laissons de côté le choix d' "étrange " pour wild, choix qui appartient à la discussion déjà mentionnée ci-dessus, et nous observons que, là où les impressions sont décrites comme "contagieuses ", le narrateur anglais avait exprimé son admiration pour les croyances et l'imagination d'Usher par le mot impressive. Le narrateur français repousse une fois de plus ce que son binôme admire, et un autre exemple de cette attitude dédaigneuse envers l'imaginaire d'Usher se trouve dans le passage suivant :

Je fus tout d'abord frappé d'une certaine incohérence [...] dans les manières de mon ami, et je découvris bientôt que cela provenait d'un 
effort incessant, aussi faible que puéril, pour maîtriser une trépidation habituelle. (Le Dantec 1951 : 342, lignes 223-225)

In the manner of my friend I was at once struck with an incoherence [...] and I soon found this to arise from a series of feeble and futile struggles to overcome a habitual trepidancy. (Mabbott 1978 : 402, lignes 170-173)

Baudelaire allait pourtant traduire futile plus tard par le sens qu'il aurait dû retenir ici, en traduisant the futility of all attempts (Mabbott 1978 : 405, ligne 260) par " la vanité de tous mes efforts " (Le Dantec 1951 : 344, ligne 329), et nous sommes donc confrontés au biais que donne Baudelaire à la relation que son narrateur entretient avec Usher, et que l'adjectif " puéril " tire vers le dédain.

Plus tard, quand les deux amis examinent les peintures qu'Usher possède, le narrateur français montre encore une fois son rejet de ces créations artistiques, et cette différence contribue aussi à créer un narrateur plus « rationnel » que celui du texte source :

Quant aux peint(u)res que couvait sa laborieuse fantaisie [...] j'essaierais vainement d'en extraire un échantillon suffisant, qui pût tenir dans le compas de la parole écrite. (Le Dantec 1951 : 345, lignes 345-346)(6)

From the paintings over which his elaborate fancy brooded [...] I would in vain endeavour to deduce more than a small portion which could lie within the compass of merely written words. (Mabbott 1978 : 405, lignes 272-273)

À part la traduction du modal would, indiquant ici habitude ou répétition dans le passé, et non un conditionnel ("j'essaierais »), il y a aussi une différence de taille entre brooding over et " couver " des objets d'art : dans la version de Baudelaire, Usher peut ainsi être pris comme le créateur de toutes ces peintures (ce qui n'est pas le cas, puisque brood over veut dire " contempler " ou " étudier quelque chose ").

(6) La version de Le Dantec présente ici une erreur en mettant " peintres ". L'erreur est corrigée dans l'édition de Claude Richard qui donne " peintures " (Richard 1989 : 412). 
Aussi, ce que le texte anglais appelle la fancy de Roderick est qualifiée de elaborate en anglais, ce qui doit se comprendre dans le sens de worked out in much detail; highly finished ou conducted with great minuteness (OED 3 : 67). Le narrateur de Baudelaire, au contraire, juge la " fantaisie » d'Usher trop « laborieuse ", que nous comprenons ici dans le sens de "compliqué, embarrassé, lourd " (Trésor 10 : 887). Il est donc clair que le narrateur français désapprouve les goûts artistiques d'Usher, là où le narrateur anglais est plutôt admiratif. Il serait donc naturel que cette attitude du narrateur français le conduise à un rejet des idées qui sont le résultat de cette "laborieuse fantaisie ».

\section{Conclusions}

Nous avons vu plusieurs passages où la traduction nous présente un narrateur plus nerveux et moins imaginatif qui, avec une confiance un peu pédante, se positionne dans une relation de plus grande intimité avec Roderick Usher. Là où le narrateur de Poe est sceptique mais intéressé et même quelque peu admiratif devant les envolées de l'imagination de son ami, celui de Baudelaire rejette son ésotérisme et son imagination artistique avec un dédain moralisant. La description de Darell Abel ci-dessous s'appliquerait alors au narrateur de Poe, mais pas à celui de Bau :

Throughout the tale, alternative explanations, natural and supernatural, of the phenomena are set forth, and we are induced by the consistently maintained device of a common-sense witness, gradually convinced in spite of his determined scepticism, to accept imaginatively the supernatural explanation. (cité dans Mabbott 1978 : 395)

Le narrateur français, quant à lui, n'est plus un témoin incarnant le rationnel, mais devient victime des événements tout comme Usher. Son scepticisme plein de bon sens est remplacé par une attitude de droiture morale, à laquelle il semble s'accrocher pour se protéger de sa propre hystérie grandissante.

L'hésitation du narrateur est aussi invalidée dans la version française par l'omission d'éléments cruciaux (dont nous n'avons ici donné que 
quelques exemples) destinés à accroître l'ambiguïté et la suspension du jugement de la part du lecteur. Le lecteur de la traduction est invité à lire le conte comme une représentation d'un événement surnaturel et irréel, et c'est exactement de cette façon que Todorov arrive à ses conclusions concernant $U_{s h e r}$ qu'il range, avec tout le reste de l'œuvre de Poe, dans la catégorie d' "étrange pur». (Todorov 1970 : 52-54).

Ainsi La chute de la maison Usher peut être lu, en suivant les concepts de Sartre, comme un conte entièrement thétique, où l'inexplicable survient comme partie intégrante de la fiction, et où il n'y a pas de suspension de la thèse. En d'autres termes, le lecteur trouve dans l'Usher de Baudelaire une allégorie lugubre sur l'extinction finale d'une lignée familiale ancestrale, sous la forme d'un conte gothique orné de symbolismes superficiels et épicé de quelques notions ésotériques. Contrairement à ce que prétend Todorov (p. 53), rien n'est vraiment expliqué rationnellement dans ce conte, et le lecteur, partageant le rejet des explications ésotériques du narrateur, quitte la scène finale en pleine perplexité. Cela semble être l'interprétation d'Éveline Pinto, qui nous parle de la version française dans les termes suivants :

Plus raisonnable que Roderick et cependant contaminé et infesté par ses affects, il est un témoin un peu trop impliqué dans la maison pour être un spectateur impartial de sa chute. De par son statut à l'intérieur de la nouvelle, il est chargé d'une narration qui a à dévoiler comme à couvrir. (Pinto 1983 : 138)

Quant au conte de Poe, il permet au lecteur, tout comme au narrateur, de s'intéresser de plus près aux envolées imaginaires de Roderick, et cela l'oblige à suspendre une thèse définitive. L'analyse que fait Kinkead-Weekes du conte indique que, dans sa version anglaise, l'histoire ne peut être lue comme un récit gothique et thétique :

The story itself tells us how to read it: not as an indulgence in the Gothick, but as an imaginatively critical exploration into the implications, the fascinations, and the price of the Gothick artist's over-development of imaginative sensationalism at the expense of body, thought, and spirit. (Kinkead-Weekes 1987:33) 
Le " sensationnalisme " et l'imagination surdéveloppée d'Usher sont présents dans la version de Baudelaire, mais les dépréciations qu'en fait le narrateur annulent la possibilité de lire le récit français comme un conte qui invite a peculiarly double kind of reading response, at once attuned to the depressive qualities of the story, yet aware that we are being asked to think and to feel more than simply 'sensation' (KinkeadWeekes 1987 : 18). Nous pouvons donc conclure avec Bieganowski, qui pointe la possibilité de lire le conte comme un rêve du narrateur :

Only at the end does the narrative focus strengthen to reveal that for the duration of the story the narrator, still standing at the tarn's edge, has been contemplating the image of the house reflected in the water. (Bieganowski 1988: 172)

Le fait que Baudelaire traduise le début de cette rêverie potentielle de façon à changer la perception et l'état contemplatif du narrateur, illustre un fois de plus combien l'impact suggestif de cette histoire, dont le début et la fin sont étroitement symétriques, peut être invalidé dans la traduction. Cela confirme qu'un glissement du mode fantastique vers le mode gothique marque bel et bien la version que Baudelaire a donnée du Usher de Poe.

wallaert@umb.u-strasbg.fr iwallaert@hotmail.com 


\section{Bibliographie}

Baudelaire, Charles. Euvres complètes II. Claude Pichois (éd.). Paris : Gallimard - Pléiade. 1976.

Berman, Antoine. Pour une critique des traductions : John Donne. Paris : "Gallimard ». 1995.

Bessière, Irène. Le récit fantastique : la poétique de l'incertain. Paris : Librairie Larousse. 1974.

Bieganowski, Ronald. " Extracts from "The Self-Consuming Narrator in Poe's Ligeia and Usher" " 1988. In May E. (éd.). 1991. Edgar Allan Poe: A Study of the Short Fiction. Boston : Twayne. 1991, pp. 164-75.

Bonaparte, Marie. Edgar Poe: Etude psychanalytique. 2 vols. Paris : Denoël \& Steele. 1933.

Cornwell, Neil. The Literary Fantastic. Londres : Harvester Wheatsheaf. 1990.

Genette, Gérard. Seuils. Paris : Éditions du Seuil. 1987.

Jackson, Rosemary. Fantasy: The literature of Subversion. Londres : Routledge. 1981.

Kinkead-Weekes, Mark. 1987. "Reflections On, and In, "The Fall of the House of Usher". " In Lee, Robert A. (éd.). Edgar Allan Poe: The Design of Order. Londres : Vision Press et Barnes \& Noble. pp. 17-34.

Le Dantec, Yves (éd.). Edgar Allan Poe: Euvres en prose. Traduction française de Charles Baudelaire. Paris : Éditions Gallimard Pléiade. 1951.

Mabbot, Thomas Oliver (éd.). Collected Works of Edgar Allan Poe: Tales and Sketches (1831-1842). Vol. 2 \& 3. Londres: Belknapp Press of Harvard University Press. 1978.

Malrieu, Joël. Le fantastique. Paris : Hachette.1992.

Meschonnic, Henri. Poétique du traduire. Paris : Verdier. 1999. 
Patterson, Arthur-S. Linfluence d'Edgar Poe sur Charles Baudelaire. Grenoble : Allier Frères. 1903.

Pinto, Éveline. Edgar Poe et l'art d'inventer. Paris : Klincksieck. 1983.

Quinn, Arthur Hobson. Edgar Allan Poe: A Critical Biography. 3e édition. Londres : Appleton Century. 1972.

Richard, Claude (éd.). Edgar Allan Poe - Contes, Essais, Poèmes. Traductions de Charles Baudelaire \& Stéphane Mallarmé, nouvelles traductions de Jean-Marie Maguin \& Claude Richard. Paris : Laffont. 1989.

Sartre, Jean-Paul. Limaginaire. Paris : Gallimard. 1940/1986.

Souiller, Didier \& Troubetzkoy, Wladimir. Littérature Comparée. 2e édition. Paris : Presse Universitaires Françaises. 1997.

Thompson, G.R. (éd.). Edgar Allan Poe: Essays and Reviews. New York : Library of America. 1984.

Todorov, Tzvetan. Introduction à la littérature fantastique. Paris : Éditions du Seuil. 1970.

Valéry, Paul. 1957. « Position de Baudelaire. » Euvres I. Jean Huytier (éd .). Paris : Gallimard - Pléiade, pp. 598-612.

Wallaert, Ineke. "The Elephant in the Dark: Corpus-based Descriptions of Translators' Style ». À paraître dans les Cahiers du GEPE, online www-umb.u-strasbg.fr/UserFiles/File/gepe, mars 2009.

Winters, Marion. «F. Scott Fitzgerald's Die Schönen un Verdammten: A Corpus-based Study of Speech-act Report Verbs as a Feature of Translators' Style. » In Meta LII, 3. pp. 412-25. 2007.

\section{Dictionnaires}

Bailly, M.A. (éd.). Dictionnaire grec-français. Paris : Hachette. 1950.

Littré, Paul Émile. Dictionnaire de la langue française. Vol 1-4. Monte Carlo : Cap. 1970. 
Oxford English Dictionary. Vol. 1-12. James A.H. Murray, Henry W. Bradley, W.A. Craigie \& C.T. Onions (éds.). Oxford : Oxford University Press. 1961.

Trésor de la langue française - Dictionnaire de la langue des XIX et XXes siècles (1789-1960). Paul Imbs (éd.), C.N.R.S., Nancy : Institut de Langue Française (vols. 1-10) \& Paris : Gallimard (vols. 11-16). 1980-1994.

Ineke Wallaert a obtenu sa licence en traduction à la Provinciale Hogeschool voor Vertalers en Tolken (École Supérieure de Traducteurs et Interprètes) de Gand en Belgique. Elle a poursuivi ses études à l'Université d'Édimbourg, où elle a obtenu un doctorat en traductologie en 2005 (Baudelaire's Rewriting of Poe : A Para-Textual Critique of the Translations). Elle a travaillé et enseigné en Belgique, Californie, Chine, Écosse et Martinique, et elle est depuis 2006 maître de conférences à l'Université de Strasbourg, où elle donne des cours de traduction et de traductologie au sein du Département des Langues étrangères appliquées. Aujourd'hui ses intérêts de recherche se portent sur les aspects philosophiques, culturels et idéologiques de la traduction, sur la traduction de la littérature pour la jeunesse, et sur les méthodes de recherche de corpus en traductologie. 